\title{
DEGRADAÇÃO ELETROQUÍMICA DA VINHAÇA USANDO ELETRODO DE DIAMANTE DOPADO COM BORO
}

\author{
Eveline Cristine Batista e Robson Tadeu Soares de Oliveira* \\ Instituto de Ciências Biológicas e Naturais, Universidade Federal do Triângulo Mineiro, 38025-180 Uberaba - MG, Brasil \\ Rafael de Queiroz Ferreira \\ Departamento de Química, Universidade Federal do Espírito Santo, Av. Fernando Ferrari, s/n, 29060-900 Vitória - ES, Brasil \\ Douglas Miwa
}

Instituto de Química de São Carlos, Universidade de São Paulo, CP 780, 13560-970 São Carlos - SP, Brasil

Mauro Coelho dos Santos

Universidade Federal do ABC, 09210-170 Santo André - SP, Brasil

Recebido em 25/10/10; aceito em 22/4/11; publicado na web em 14/6/11

\begin{abstract}
ELECTROCHEMICAL OXIDATION OF VINASSES USING A BORON DOPED DIAMOND ELECTRODE. The degradation of vinasses in aqueous solution from ethanol industry has been investigated by electrochemical oxidation using a boron doped diamond electrode (BDD). Samples of vinasses were electrolyzed in medium of $\left(0.1 \mathrm{~mol} \mathrm{~L}^{-1}\right) \mathrm{Na}_{2} \mathrm{SO}_{4}$ solutions at controlled potentials of +2.4 , +3.0 and $+4.0 \mathrm{~V}(v s . \mathrm{Ag} / \mathrm{AgCl})$ and exhibited considerable reduction of total organic carbon. The cyclic voltammetry studies indicate that the vinasses are oxidized irreversibly over the $\mathrm{BDD}$ at $2.0 \mathrm{~V}(v \mathrm{~s} . \mathrm{Ag} / \mathrm{AgCl})$ in diffusion controlled process. From the experimental results it is clear that the BDD electrode can be a valuable tool to the electrochemical degradation of vinasses in practical applications.
\end{abstract}

Keywords: vinasses; electrochemical degradation; boron doped diamond electrode.

\section{INTRODUÇÃO}

Diante da necessidade de minimizar os impactos causados por diversos poluentes orgânicos, há um grande interesse no desenvolvimento de métodos eficientes para degradação de diferentes classes de substâncias. Estes métodos visam reduzir ao máximo a complexidade química das moléculas, assim como os níveis de resíduos gerados. ${ }^{1-23}$ Dentre os poluentes que causam danos ao meio ambiente pode-se destacar a vinhaça, que é produzida em grande escala no Brasil devido à produção de etanol, setor que está em plena expansão, em virtude da necessidade da substituição de combustíveis fósseis pelos biocombustíveis.

A vinhaça é caracterizada como um efluente de destilarias com alto poder poluente e alto valor fertilizante. É composta principalmente por ácido acético e lático, glicerol, açúcares, alta concentração de sais minerais e matéria orgânica. Geralmente é convertida em adubo, entretanto, quando descartada no meio ambiente causa graves problemas ambientais. ${ }^{4,5}$ Sua força poluente, cerca de cem vezes a do esgoto doméstico, decorre da sua riqueza em matéria orgânica, além de possuir três importantes componentes: nitrogênio, fósforo e potássio. A poluição dos cursos d'água é caracterizada pela introdução em sua massa líquida de qualquer elemento que lhe seja estranho. Mesmo que esse elemento se constitua de alguma riqueza, a água que o transporta estará poluída. Por muito tempo a vinhaça foi simplesmente descartada nos rios, poluindo-os consideravelmente. ${ }^{6}$ Geralmente, efluentes provenientes de destilarias são tratados por meio de métodos biológicos (aeróbicos e anaeróbicos), ou por meio de tratamentos físico-químicos, como adsorção, coagulação e floculação, oxidação, tratamento utilizando membranas e evaporação/combustão. ${ }^{7}$

Os métodos eletroquímicos surgem como uma alternativa promissora para esses fins, pois permitem a transformação prévia de

*e-mail: robson@icbn.uftm.edu.br certos compostos orgânicos em substâncias biodegradáveis ou levam, eventualmente, à formação de $\mathrm{CO}_{2}+\mathrm{H}_{2} \mathrm{O}$ ("incineração eletroquímica"). A grande vantagem destes métodos é que o reagente principal é o elétron e os resíduos adicionais gerados são mínimos. Estudos mostraram que compostos orgânicos de interesse ambiental podem ser eletro-oxidados eficientemente em soluções aquosas, utilizandose eletrodos de óxidos mistos de Ti, Ru e Sn. ${ }^{8}{ }^{8}$ Uma opção que vem sendo testada há alguns anos é a utilização de eletrodos de óxidos metálicos, tais como $\mathrm{PbO}_{2}$ e $\mathrm{SnO}_{2} \mathrm{e},{ }^{10}$ mais recentemente, $\mathrm{RuO}_{2} \mathrm{e}$ $\mathrm{IrO}_{2},{ }^{11,12}$ entre outros. A estabilidade química e eletroquímica desses materiais anódicos pode ser satisfatória, dependendo das condições experimentais empregadas. Entretanto, para meios mais agressivos e potenciais muito elevados, a eficiência destes materiais pode ser comprometida.

Na oxidação anódica, os poluentes orgânicos são destruídos pelos radicais hidroxilas gerados como intermediários da oxidação da água na superfície dos ânodos, como apresentado pela Equação 1:

$$
\mathrm{M}+\mathrm{H}_{2} \mathrm{O} \rightarrow \mathrm{M}\left({ }^{\circ} \mathrm{OH}\right)+\mathrm{H}^{+}+\mathrm{e}^{-}
$$

onde $\mathrm{M}\left({ }^{\bullet} \mathrm{OH}\right)$ denota o radical hidroxila na superfície do ânodo $M . \cdot^{13,14}$

A grande vantagem de se utilizar estes métodos para a degradação de poluentes orgânicos está na não seletividade dos radicais hidroxilas. Neste caso, moléculas orgânicas são desidrogenadas ou hidroxiladas até a completa mineralização (conversão em $\mathrm{CO}_{2}$, água e íons inorgânicos).

As propriedades eletroquímicas do eletrodo de diamante dopado com boro (DDB) são significativamente diferentes daquelas apresentadas pelos outros alótropos do carbono. Deste modo, o DDB apresenta características muito interessantes, em comparação com materiais eletródicos como grafite pirolítico e carbono vítreo, ${ }^{15}$ como uma grande janela eletroquímica, alta estabilidade, resistência em meios agressivos e a eletrogeração de radicais hidroxilas (forte agente 
oxidante), ${ }^{16}$ que faz deste material um forte candidato para o estudo da oxidação destrutiva de compostos orgânicos aromáticos, ${ }^{17-18192021}$ como mostrado na Equação 2:

$$
\mathrm{DDB}\left(\mathrm{H}_{2} \mathrm{O}\right) \rightarrow \mathrm{DDB}(\cdot \mathrm{OH})+\mathrm{H}^{+}+\mathrm{e}^{-}
$$

Uma ampla revisão das aplicações correntes do DDB, em eletroquímica, foi feita por Kraft. ${ }^{22}$ Nesta revisão, o autor abordou os diferentes tipos de eletrodos de diamante e seus métodos de preparação e, posteriormente, suas propriedades eletroquímicas em diferentes eletrólitos. Também foram abordadas estratégias de modificações para o DDB dopado, seguidas de suas aplicações práticas em eletrossíntese, eletroanálise, conversão de energia e biotecnologia.

Diante das potencialidades do DDB, frente à combustão eletroquímica de poluentes orgânicos, Oliveira et al. ${ }^{14}$ investigaram a oxidação eletroquímica do benzeno $\left(10^{-2} \mathrm{~mol} \mathrm{~L}^{-1}\right)$ em meio de $\mathrm{H}_{2} \mathrm{SO}_{4}$ $\left(0,5 \mathrm{~mol} \mathrm{~L}^{-1}\right)$. Foram realizados experimentos voltamétricos e de eletrólises a potenciais controlados. $\mathrm{O}$ benzeno mostrou um pico de oxidação em aproximadamente 2,0 V (vs. Ag/AgCl). Após 5 h de eletrólise, os autores constataram a total mineralização do benzeno, que foi confirmada por meio de medidas de teor de carbono orgânico total. Outros trabalhos também mostraram a eficiência da utilização do DDB para combustão eletroquímica. Foi estudada a oxidação eletroquímica do 4-clorofenol em meio ácido sobre o DDB, utilizando as técnicas de voltametria cíclica, cronoamperometria e eletrólises exaustivas a altos potenciais anódicos. ${ }^{23,24}$ Os autores observaram a incineração completa do 4-clorofenol sobre o DDB por meio dos radicais hidroxilas eletrogerados na superfície. Inesta et al. ${ }^{21}$ reportaram a oxidação destrutiva de fenol sobre um eletrodo de DDB realizada em diferentes regiões de potenciais e observaram que para a região de potencial antes do desprendimento de oxigênio ocorre a transferência direta de elétrons, formando um filme polimérico na superfície do eletrodo.

Considerando as propriedades eletroquímicas dos eletrodos de DDB, já mencionadas, com relação à degradação de poluentes orgânicos, foi proposta a degradação da vinhaça. Foram realizados experimentos usando voltametria cíclica, eletrólises a potenciais controlados e, posteriormente, análise do teor de carbono orgânico total.

\section{PARTE EXPERIMENTAL}

Os DDB foram adquiridos do Centro Suíço de Eletrônica e Micro-técnica SA (CSEM), Neuchatêl, Suíça. O teor de boro final dos eletrodos empregados foi da ordem de 8000 ppm. Todos os experimentos eletroquímicos foram realizados utilizando uma EG\&G Princeton PAR potenciostato/galvanostato modelo 273A.

Os experimentos eletroquímicos foram realizados usando uma célula eletroquímica de três compartimentos (vidro Pyrex ${ }^{\circledR}$ ) com entrada para deaeração. Os eletrodos de trabalho empregados foram os DDB com área de $0,2 \mathrm{~cm}^{2}$. O eletrodo de referência foi $\mathrm{Ag} / \mathrm{AgCl}$ e todos os potenciais são referentes a este eletrodo. O eletrodo auxiliar foi uma placa de Pt de $2 \mathrm{~cm}^{2}$. Antes do uso, a superfície do eletrodo DDB foi limpa com álcool e lavada com água ultrapura. O eletrólito utilizado foi adquirido da Merck $\left(\mathrm{Na}_{2} \mathrm{SO}_{4}, \mathrm{P} . \mathrm{A}\right)$. A água para preparação de soluções foi purificada pelo sistema Milli-Q da Millipore ${ }^{\circledR}$. As medidas eletroquímicas foram realizadas empregando-se soluções eletrolíticas de $\mathrm{Na}_{2} \mathrm{SO}_{4} 0,1 \mathrm{~mol} \mathrm{~L}{ }^{-1}(20,0 \mathrm{~mL})$, contendo vinhaça (2,0 mL). A vinhaça foi coletada na usina Santa Cruz S/A Açúcar e Álcool, em Araraquara - SP, usando-se um frasco previamente limpo. A oxidação eletroquímica da vinhaça foi investigada em diferentes potenciais e a cinética de degradação foi avaliada por meio de um espectofotômetro UV-Vis, modelo SPECTRUM SP - 2000 UV, e um analisador de carbono orgânico total Shimadzu TOC-VCPH.

\section{RESULTADOS E DISCUSSÃO}

Inicialmente, foram realizados estudos eletroquímicos visando determinar a eletroatividade da vinhaça frente ao DDB. Deste modo, o DDB empregado recebeu um pré-tratamento anódico de $+3,0 \mathrm{~V}$ (vs. $\mathrm{Ag} / \mathrm{AgCl}$ ) durante $300 \mathrm{~s}$, em meio de $\mathrm{H}_{2} \mathrm{SO}_{4}\left(0,1\right.$ mol.L $\left.{ }^{-1}\right)$, com o objetivo oxidar possíveis espécies adsorvidas sobre a superfície eletródica. A anodização do DDB também teve como objetivo aumentar a janela de potenciais no sentido anódico. Esse comportamento eletroquímico do DDB foi previamente reportado na literatura. ${ }^{25-2627}$ Após o tratamento anódico, voltamogramas cíclicos realizados somente no eletrólito de suporte $\left(0,1 \mathrm{~mol} \mathrm{~L}^{-1} \mathrm{Na}_{2} \mathrm{SO}_{4}\right)$ e da amostra contendo vinhaça foram registrados (Figura 1).

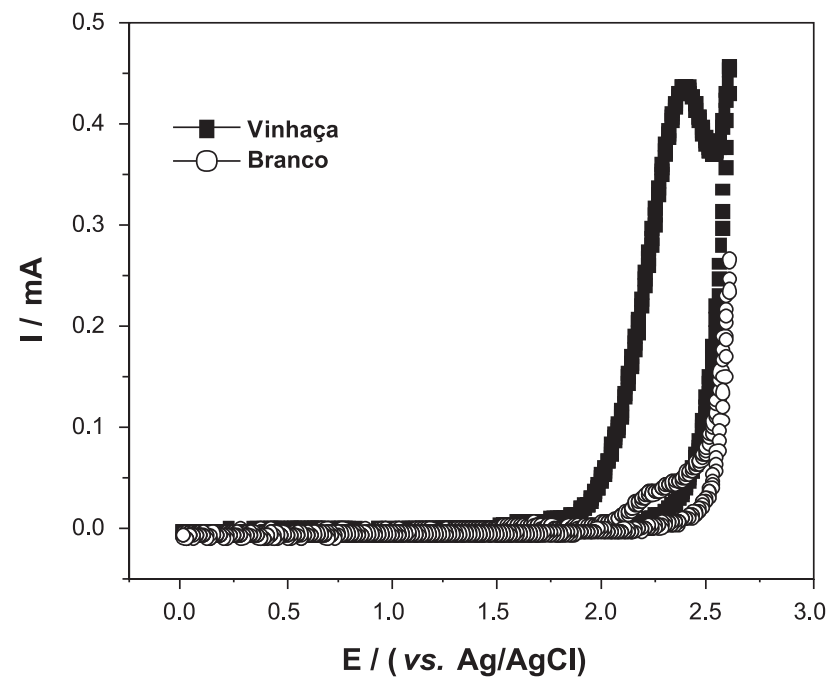

Figura 1. Voltamogramas cíclicos em meio de $\mathrm{Na}_{2} \mathrm{SO}_{4} \mathrm{O}, 1 \mathrm{~mol} \mathrm{~L} \mathrm{~L}^{-1}$ (linha tracejada) e para a vinhaça $\left(2,0 \mathrm{~mL}\right.$ ) (linha sólida) em meio de $\mathrm{Na}_{2} \mathrm{SO}_{4} \mathrm{O}, 1$ mol $L^{-1}(20,0 \mathrm{~mL})$ obtidos sobre o DDB. Velocidade de varredura $0,1 \mathrm{~V} \mathrm{~s}^{-1}$

Durante a varredura anódica (Figura 1), entre 0 e 2,6 V (vs. Ag/ $\mathrm{AgCl}$ ), um pico relacionado à oxidação eletroquímica dos componentes presentes na vinhaça foi observado em aproximadamente 2,4 V. Neste caso, o pico observado está associado a um processo irreversível. Foram realizados experimentos variando-se a velocidade de varredura, observando-se uma relação linear entre I $p s . v^{1 / 2}$ (Figura 2), indicando que a oxidação eletroquímica da vinhaça é representada por um processo controlado por difusão. ${ }^{28}$

A degradação da vinhaça foi, em seguida, investigada por meio de eletrólises a potenciais controlados. Foram eletrolisadas soluções $0,1 \mathrm{~mol} \mathrm{~L}^{-1}$ de $\mathrm{Na}_{2} \mathrm{SO}_{4}(20,0 \mathrm{~mL})$ contendo vinhaça $(2,0 \mathrm{~mL})$. Os potenciais escolhidos preliminarmente para a realização das eletrólises foram $+2,4 \mathrm{e}+3,0 \mathrm{~V}$ ( $v$ s. $\mathrm{Ag} / \mathrm{AgCl}$ ), respectivamente. $\mathrm{O}$ potencial de $+2,4 \mathrm{~V}$ foi escolhido devido ser exatamente o potencial onde foi observada a oxidação da vinhaça, ou seja, no potencial de pico. A investigação do processo de combustão eletroquímica da vinhaça, em $+3,0 \mathrm{~V}$, foi realizada devido este valor de potencial se localizar após o pico de oxidação. Neste caso, observou-se que em +3,0 V começou também a ocorrer desprendimento de oxigênio na interface eletródica. Deste modo, foram analisadas as oxidações da vinhaça no pico e após o pico de oxidação. Alíquotas de $1,0 \mathrm{~mL}$ das soluções eletrolisadas foram coletadas a cada $15 \mathrm{~min}$, durante 60 min e analisadas por meio de medidas de UV-Vis, em $254 \mathrm{~nm}$ (absorção máxima observada). O valor de absorbância máxima observada está de acordo com medidas previamente realizadas por Beltrán et al.,${ }^{29}$ que investigaram a degradação de águas residuárias de destilarias juntamente com esgoto doméstico, usando a ozoniza- 


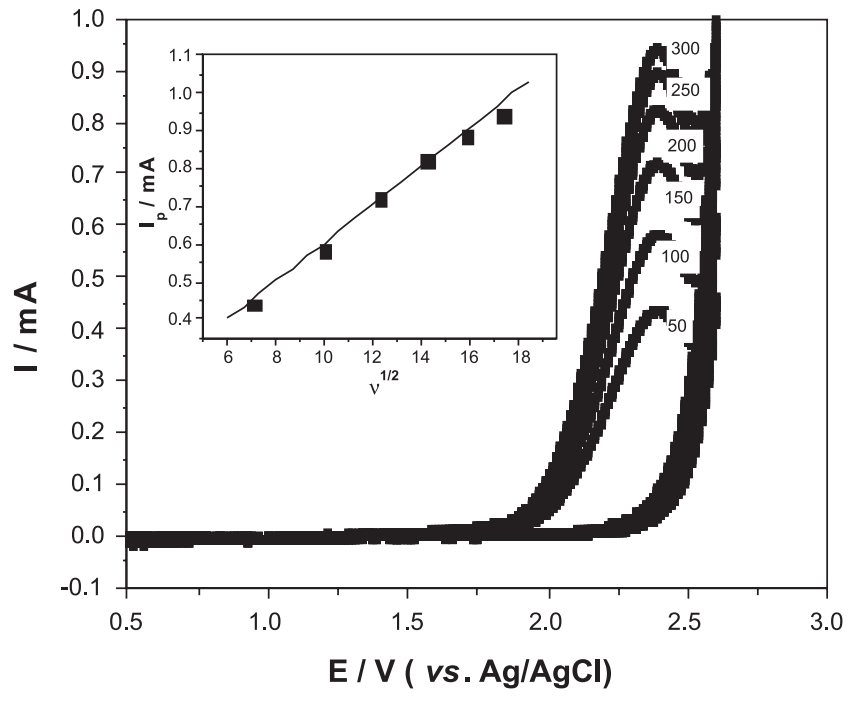

Figura 2. (a) Voltamogramas cíclicos obtidos para a vinhaça $(2,0 \mathrm{~mL}) \mathrm{em}$ meio de $\mathrm{Na}_{2} \mathrm{SO}_{4} 0,1 \mathrm{~mol} \mathrm{~L}^{-1}(20,0 \mathrm{~mL})$ sobre o eletrodo de DDB em função da velocidade de varredura; (b) Ip vs. $v^{1 / 2}$ para vinhaça em meio de $\mathrm{Na}_{2} \mathrm{SO}_{4}$ $0,1 \mathrm{~mol} \mathrm{~L}^{-1}$ sobre o DDB

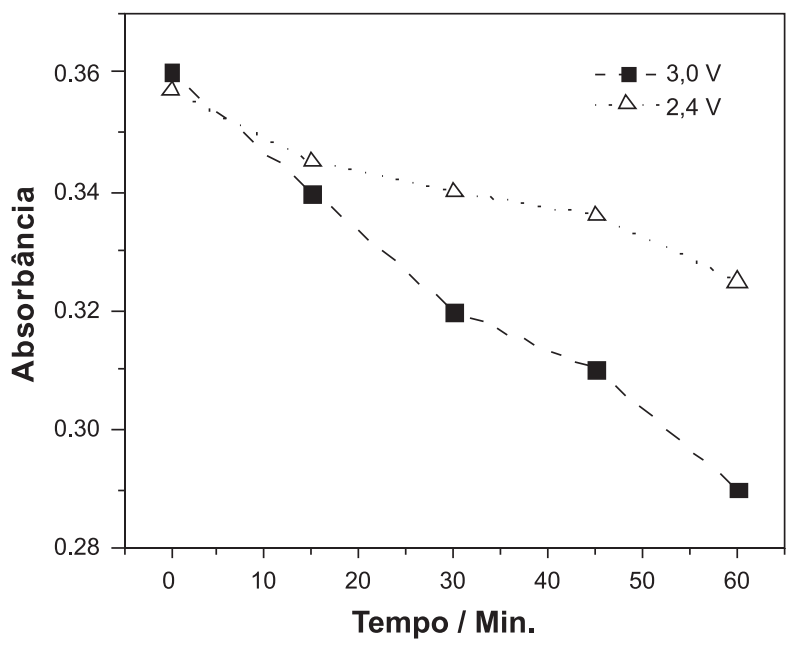

Figura 3. Espectros de UV-Vis registrados em $254 \mathrm{~nm}$ para vinhaça eletrolisada em $+2,4$ e +3,0 V (vs. Ag/AgCl) em função do tempo

ção. Os valores de absorbância versus o tempo de eletrólise estão apresentados na Figura 3.

Os espectros de UV-Vis mostraram que há um decréscimo nos valores de absorbância em função do tempo de eletrólise. Esse decréscimo se mostrou mais acentuado na eletrólise realizada em $+3,0$ $\mathrm{V}$ (vs. $\mathrm{Ag} / \mathrm{AgCl}$ ). Nesse caso, a reação de desprendimento de oxigênio contribuiu positivamente no processo de oxidação da vinhaça.

Nota-se que as absorbâncias registradas em $+2,4 \mathrm{~V}$ apresentaram comportamentos praticamente lineares, em função do tempo de eletrólise ( $60 \mathrm{~min}$ ). Para o potencial de $+3,0 \mathrm{~V}$, observou-se um comportamento semelhante.

O tratamento eletroquímico de águas residuárias contendo espécies orgânicas tem sido encontrado na literatura relatando a utilização do eletrodo de DDB no estudo de algumas destas espécies, levando à combustão eletroquímica, gerando compostos menos tóxicos, podendo chegar a $\mathrm{CO}_{2}$ e $\mathrm{H}_{2} \mathrm{O} .^{30,31}$ Troster et al..$^{32}$ reportaram o tratamento e desinfecção de águas residuárias utilizando eletrodos de DDB em processos oxidativos em altos potenciais via formação dos radicais hidroxilas. As vantagens obtidas ao se usar o eletrodo de DDB em altos potenciais para essa finalidade foram descritas como alta esta- bilidade, inércia química e longo tempo de vida útil do eletrodo. Os resultados demonstraram uma eficiência de corrente da ordem de $90 \%$. Diante da possibilidade de se usar potenciais elevados, levando a uma maior eficiência do processo, a degradação da vinhaça também foi realizada em $+4,0 \mathrm{~V}$ ( $v$ s. $\mathrm{Ag} / \mathrm{AgCl}$ ). $\mathrm{O}$ potencial de $+4,0 \mathrm{~V}$ foi escolhido visando avaliar a influência do aumento de potencial (na região de desprendimento de oxigênio) na degradação do poluente investigado. Os valores de potenciais empregados nesse trabalho não são alcançados por eletrodos como grafite pirolítico ou carbono vítreo, devido a suas pequenas janelas eletroquímicas, comparadas com a do DDB. ${ }^{33}$ Deste modo, foram realizadas eletrólises nos potenciais de $+2,4,+3,0$ e $+4.0 \mathrm{~V}$, respectivamente. A degradação da vinhaça foi, posteriormente, seguida por meio de análise de TOC.

Nesses experimentos, utilizou-se a mesma metodologia adotada nas eletrólises anteriores. Entretanto, as alíquotas coletadas foram diluídas em 9,0 mL de água destilada, para posterior determinação do teor de carbono orgânico total. Cada alíquota foi coletada a cada $15 \mathrm{~min}$. Esses experimentos foram conduzidos durante $60 \mathrm{~min}$. Os valores de TOC versus o tempo de eletrólise estão apresentados na Figura 4.

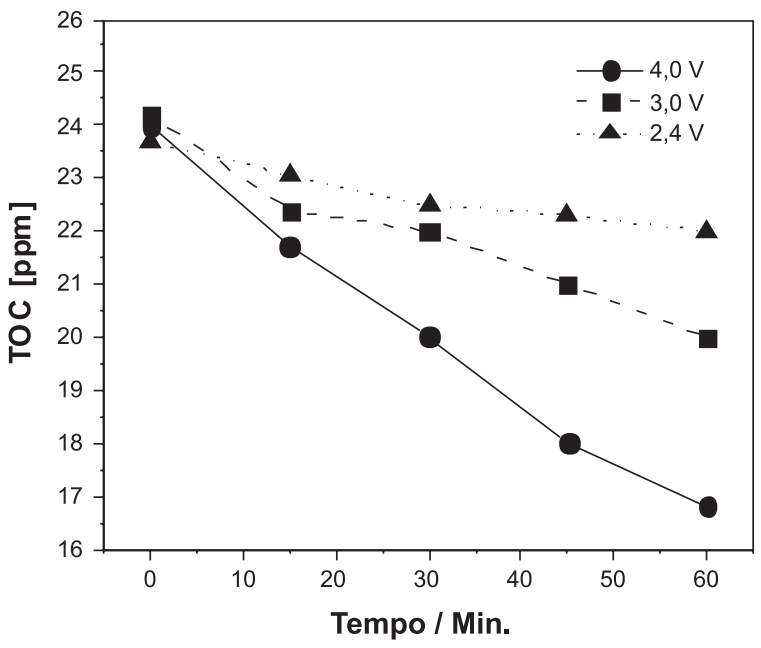

Figura 4. Valores determinados do teor de carbono orgânico total durante as eletrólises realizadas usando 2,4; 3,0 e 4,0 V (vs. $\mathrm{Ag} / \mathrm{AgCl})$ até um tempo final de $60 \mathrm{~min}$

Na Figura 4 observa-se que houve uma redução significativa do TOC em função do aumento do potencial. Neste caso, com o aumento do potencial, observou-se um acréscimo da mineralização da matéria orgânica contida na vinhaça, devida ao aumento de radicais hidroxilas formados sobre o DDB (Equação 2). Em ambos os experimentos de eletrólise, ao longo de $60 \mathrm{~min}$, observou-se um comportamento praticamente linear entre TOC vs. tempo. Esse comportamento linear também foi observado pelas medidas de UV-Vis (absorbância vs. tempo).

Visando determinar o tempo para a total mineralização do poluente orgânico investigado, outros experimentos foram conduzidos durante $5 \mathrm{~h}$. Neste caso, as alíquotas de 1,0 $\mathrm{mL}$ foram coletadas a cada hora e diluídas também em 9,0 mL de água deionizada, para posterior análise de TOC. As eletrólises foram realizadas em $+3,0 \mathrm{e}$ $+4,0 \mathrm{~V}$ (vs. $\mathrm{Ag} / \mathrm{AgCl}$ ). Esses valores de potenciais foram escolhidos mediante o decréscimo do teor de carbono orgânico total observado nos experimentos anteriores. Os valores de TOC em função do aumento do potencial estão mostrados na Figura 5.

Nota-se ao longo de $5 \mathrm{~h}$ de eletrólise, o TOC sofreu um decréscimo bastante acentuado, em ambos os potenciais investigados. Especificamente em $+4,0 \mathrm{~V}$, observa-se um decaimento exponencial, 


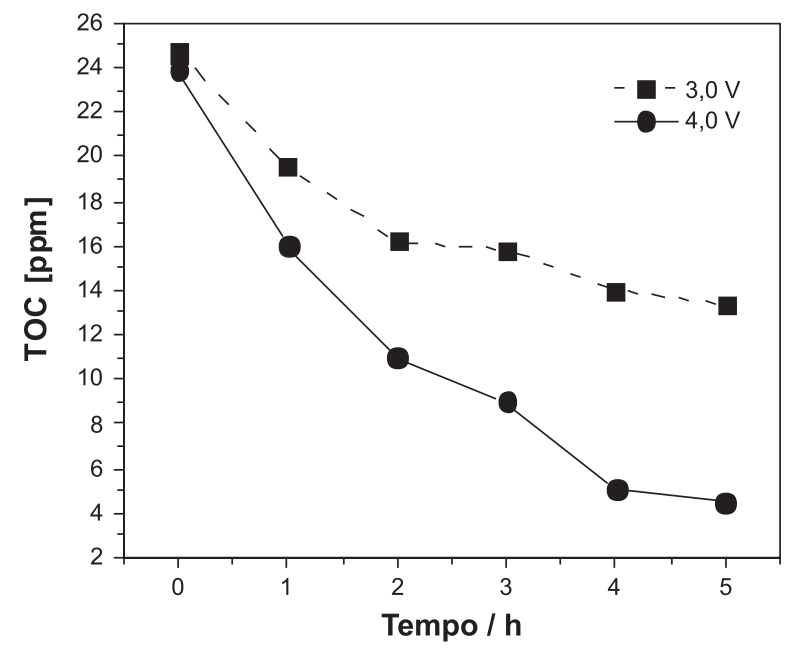

Figura 5. Valores determinados do teor de carbono orgânico total durante as eletrólises realizadas usando 3,0 e 4,0 V (vs. Ag/AgCl) até um tempo final de $5 \mathrm{~h}$

onde a partir de $4 \mathrm{~h}$ de eletrólise a vinhaça foi consideravelmente mineralizada, restando somente $4 \mathrm{ppm}$.

Estudos realizados por Beltrán et al., ${ }^{29}$ visando a degradação da vinhaça, usando a ozonização, não se mostraram viáveis, uma vez que há a necessidade de utilizar grande quantidade de ozônio seguido de um controle rígido de $\mathrm{pH}$. Neste sentido, os autores observaram baixos valores de remoção do teor de matéria orgânica.

Diante da dificuldade da remoção da matéria orgânica presente na vinhaça usando a ozonização, o eletrodo de diamante dopado com boro mostrou-se eficiente para a oxidação eletroquímica da vinhaça, uma vez que a remoção do teor de carbono orgânico total ao longo de $5 \mathrm{~h}$ de eletrólise foi de aproximadamente 85\%. É importante salientar que essa amostra apresenta grande complexidade em função da sua composição química. Deste modo, o DDB apresentou vantagem sobre outros materiais anódicos, que podem sofrer corrosão em elevados potenciais ao longo do tempo de eletrólise, o que possibilitou a investigação da degradação do poluente orgânico em $+4,0 \mathrm{~V}$. Os valores de TOC obtidos nos experimentos estão de acordo com valores máximos estabelecidos pela Resolução CONAMA Nº 357, de 17/3/2005, cujos valores de TOC para águas salobras e salinas são 3, 5 e 10 ppm, para águas das classes 1,2 e 3 , respectivamente. ${ }^{34}$

\section{CONCLUSÃO}

A oxidação eletroquímica operando em potenciais de $+3,0$ e $+4,0 \mathrm{~V}$, utilizando o DDB como ânodo, mostrou-se um método bastante eficiente para promover a degradação da vinhaça dissolvida em solução aquosa de $\mathrm{Na}_{2} \mathrm{SO}_{4}$. $\mathrm{O}$ tratamento da solução contendo aproximadamente $24 \mathrm{ppm}$ de carbono orgânico total levou a uma redução de $57 \%$ deste teor, quando a eletrólise foi realizada em $+3,0 \mathrm{~V}$ (durante $5 \mathrm{~h}$ ). Utilizando-se o potencial de $+4,0 \mathrm{~V}$ a redução do TOC foi de aproximadamente $85 \%$. O método foi aplicado com sucesso no tratamento do efluente coletado na usina Santa Cruz S/A Açúcar e Álcool, em Araraquara - SP.

\section{AGRADECIMENTOS}

Ao CNPq (Proc. 119200/20082), à CAPES e FAPEMIG pelo suporte financeiro.

\section{REFERÊNCIAS}

1. Flox, C.; Cabot, P. L.; Centellas, F.; Garrigo, J. A.; Rodrigues, R. M.; Arias, C.; Brillas, E.; Chemosphere 2006, 6, 892.

2. Brillas, E.; Baños, M. A.; Skoumal, M.; Cabot, P. L.; Garrido, J. A.; Rodríguez, R. M.; Chemosphere 2007,68, 199.

3. http://www.epa.gov/oppfead1/international/pops.htm, acessada em Outubro 2010.

4. Shojasadati, S. A.; Khalilzadeb, R.; Jalilzadeh, A.; Sanaei, H. R.; Resour. Conserv. Recy. 1999, 27, 125.

5. Jimenez, A. M.; Borja, R.; Martin, A.; Process Biochem. 2003, 38, 1275.

6. Cabello, P. E.; Scognamiglio, P. F.; Terán, F. J. C.; Engenharia Ambiental - Espírito Santo do Pinhal 2009, 6, 321.

7. Sowmeyan, R.; Swaminathan, G.; J. Hazard. Mater. 2008, 152, 453.

8. Pelegrino, R. L.; Iglia, R. A.; Sanches, C. G.; Avaca, L. A.; Bertazzoli, R.; J. Braz. Chem. Soc. 2002, 13, 60.

9. Panizza, M.; Ouattara, L.; Baranova, E.; Comninellis, C.; Electrochem. Commun. 2003, 5, 365.

10. Johnson, D. C.; Feng, J.; Houk, L. L.; Electrochim. Acta 2000, 46, 323.

11. Kim, K. W.; Lee, E. H.; Kim, J. S.; Shin, K. H.; Jung, B. I.; J. Electrochem. Soc. 2002, 149, D187.

12. Rajkumar, D.; Palanivelu, K.; Ind. Eng. Chem. Res. 2003, 42, 1833.

13. Panizza, M.; Cerisola, G.; Electrochim. Acta 2005, 51, 191.

14. Oliveira, R. T. S.; Salazar-Banda, G. R.; Santos, M. C.; Calegaro, M. L.; Miwa, D. W.; Machado, S. A. S.; Avaca, L. A.; Chemosphere 2007, 66, 2152.

15. Pleskov, Y.; Russ. J. Electrochem. 2002, 38, 1275.

16. Marselli, B.; Garcia-Gomes, J.; Michaud, P. A.; Rodrigo, M. A.; Comninellis, C.; J. Electrochem. Soc. 2003, 150, D79.

17. Flox, C.; Antonio, G. J.; Marı, R. R.; Centella, F. S.; Cabo, P. T.; Conchita, A.; Brillas, E.; Electrochim. Acta 2005, 50, 3685.

18. Cañizares, P.; Sáez, C.; Lobato, J.; Rodrigo, M. A.; Electrochim. Acta 2004, 49, 4641

19. Morão, A.; Lopes, A.; Pessoa de Amorim, M. T.; Gonçalves, I. C.; Electrochim. Acta $2004,49,1587$.

20. Codognoto, L.; Machado, S. A. S.; Avaca, L. A.; J. Appl. Electrochem. 2003, 33, 951.

21. Iniesta, J.; Michaud, P. A.; Panizza, M.; Cerisola, G.; Aldaz, A.; Comninellis, Ch.; Electrochim. Acta 2001, 46, 3573.

22. Kraft, A.; Int. J. Electrochem. Sci. 2007, 2, 355.

23. Rodrigo, M. A.; Michaud, P. A.; Duo, I.; Panizza, M.; Cerisola, G.; Comninellis, C.; J. Electrochem. Soc. 2001, 148, D60.

24. Gherardini, L.; Michaud, P. A.; Panizza, M.; Comninellis, C.; Vatistas, N.; J. Electrochem. Soc. 2001, 148, D78.

25. Salazar-Banda, G. R.; Suffredini, H. B.; Calegaro, M. L.; Tanimoto, S. T.; Avaca, L. A.; J. Power Sources 2006, 162, 9.

26. Oliveira, R. T. S.; Salazar-Banda, G. R.; Oliveira, S. C.; Ferreira, V. S.; Avaca, L. A.; Electroanalysis 2007, 19, 1189.

27. Oliveira, R. T. S.; Salazar-Banda, G. R.; Machado, S. A. S.; Avaca, L. A.; Electroanalysis 2008, 20, 396.

28. Bard, A.; Faulkner, L. R.; Electrochemical Methods. Fundamentals and Applications, $2^{\text {nd }}$ ed., John Wiley \& Sons: Danvers, 2000.

29. Breltrán, F.; Garcia-Araya, J. F.; Alvarez, P. M.; J. Agric. Food Chem. 1999, 47, 3911.

30. Foord, J. S.; Holt, K. B.; Compton, R. G.; Marken, F.; Diamond Relat. Mater. 2001, 10, 662.

31. Chen, X.; Chen, G.; Yue, P. L.; Chem. Eng. Sci. 2003, 58, 995.

32. Troster, I.; Schafer, L.; Fryda, M.; Matthee, T.; Water Sci. Technol. 2004, 49, 207.

33. Nossol E.; Dissertação de Mestrado, Universidade Federal do Paraná, Brasil, 2009.

34. http://www.cetesb.sp.gov.br/Agua/praias/res_conama_357_05.pdf, acessada em Agosto 2010. 